\title{
Identifying Strategies for Managing Critical Success Factors of BPM initiatives in Brazilian Public Organizations
}

\author{
Higor M. Santos ${ }^{1}$, Carina F. Alves ${ }^{1}$, George V. Santos ${ }^{1}$ and André L. Santana ${ }^{1}$ \\ ${ }^{1}$ Center of Informatics, Federal University of Pernambuco \\ Post Office 50.740-560 - Recife - PE - Brasil \\ \{hrms,cfa,gavs,afls2\}@cin.ufpe.br
}

\begin{abstract}
Business Process Management involves theoretical and operational elements from different areas, being a multidisciplinary field. In previous studies, we identified critical success factors of BPM initiatives in Brazilian Public Organizations. In this work, we intend to investigate how to manage these factors. To achieve this goal, we performed a focus group with five professionals with experience in BPM initiatives within the public sector. The main contribution of this study is to fill the gap in the literature concerning critical success factors for BPM initiatives in public organizations.
\end{abstract}

\section{Introduction}

Organizational goals are perceived in the execution of activities that add value to the institution and its customers (Kotler \& Armstrong, 2009). In the context of private organizations, this value generation essentially corresponds to profit, market share and brand growth. For public organizations or non-profit institutions, the leading priority is to provide citizens with services of quality excellence and that require minimum resource consumption. The constant search for achieving strategic objectives and maximizing return on investment reinforces the relevance of progressively improving business processes. The introduction of Business Process Management (BPM) practices shall pave the way for the alignment between strategic areas and functional sectors.

The constant search for achieving strategic objectives emphasizes the importance for organizations to continuously improve their business processes. With the introduction of Business Process Management (BPM) approaches and concepts, the alignment between strategic areas and functional sectors becomes possible. In addition, organizations are provided with a solid structure, which supports quicker and more appropriate answers to changes in the external environment.

BPM was developed from a variety of management approaches, including Toyota Production System (TPS), Total Quality Management (TQM), Business Process Reengineering (BPR) and Six Sigma. Nevertheless, there is a need for technical standards which provide a precise definition of process management, modelling notation, implementation guide, maturity model, among other aspects associated with BPM discipline (ABPMP, 2009); (Jeston \& Nelis, 2008); (Recker, et al., 2009); (Weske, 2007). The inconsistence of patterns and conceptual understandings regarding BPM may be primary causes for the large number of initiatives that failed in the past.

In this scenario, the motivation for this work is threefold. First, we want to understand the reasons behind the high failure rates of BPM initiatives (Grover, 1999; 
Trkman, 2010). Second, we identified a short number of empirical studies about factors that may help BPM initiatives to succeed or that may lead them to fail (Bandara et al. 2005). Finally, we aim to analyze the particularities of the public sector regarding the implementation of BPM initiatives (Gulledge \& Sommer, 2002; Houy et al. 2010). By investigating the adoption of BPM by Brazilian public sector, we aim to elicit factors that are critical for the success of their BPM initiatives. In addition, we intend to discuss strategies to manage these factors.

Considering studies from (Aparecida da Silva et al. 2012; Paim et al. 2008), our hypothesis is that for BPM projects to thrive it is essential to appropriately manage these factors and thereby mitigate failure probabilities. Although there are several studies investigating Critical Success Factors (CSF) of BPM initiatives (ABPMP, 2009; Burlton, 2011; Jeston \& Nelis (2008); Trkman, 2010), these are mainly centered on private organizations. To address this research gap, we conducted an empirical research to answer the following Research Questions (RQ):

RQ1: What are the CSF of BPM initiatives identified in the literature?

RQ2: What are the CSF evidenced in BPM initiatives in the public sector?

RQ3: How to manage CSF evidenced in BPM initiatives in the public sector?

We previously discussed RQ1 and RQ2 in Santos et al. (2011). This paper focuses on analyzing RQ3. This paper is structured as follows: Section 2 presents the background of this research. Section 3 presents the research methods used to conduct the empirical study. Section 4 describes study results. Finally, Section 5 concludes the paper and provides directions for future research.

\section{Business Process Management}

The evolution achieved by organizations since the end of XIX century, with the emergence of new organizational models, is providing a novel collaborative way of work (Ko, et al., 2009). This happens within the organization (between its unities and departments) as well as within its relationships (networked organizations). Another remarkable characteristic of this evolution is the increasing focus on the quality of the product and/or service delivered to the customer, which is constructed via approaches centred on a business process perspective.

Business processes are procedures which characterize an organization's operation and are supported by internal processes. They result in a product or service perceived by an external client (Jeston \& Nelis, 2008). Given their importance within the organizational environment, Business Process Management (BPM) acts as a discipline which aims at managing and aligning the main organizational activities with its strategic goals.

The Gartner Group claims that BPM is a management approach that treats business processes as assets directly contributing to the organizational performance, leading to an operational excellence and to business agility (McCoy, 2011). In its turn, the Object Management Group (OMG) considers BPM as a set of techniques focused on a continuous and interactive improvement of an organization business processes (OMG, 2010). Similarly, (Jeston and Nelis, 2008) claims that BPM is the accomplishment of 
organizational goals through the improvement, management and control of essential business processes.

The adoption of BPM concepts and practices involves activities such as identifying, modelling, executing, measuring, monitoring and improving business processes, achieving all organizational levels. According to Sentanin, Santos and Jabbour (2008), identifying and managing the CSF associated with BPM initiatives can increase their chances of success. These factors may vary among organizations and business segments. Given that, researchers and consultants have tried to list groups of CSF which generally figure out in BPM initiatives.

\section{Research Methods}

The methods that supported this study are following presented (Figure 1).

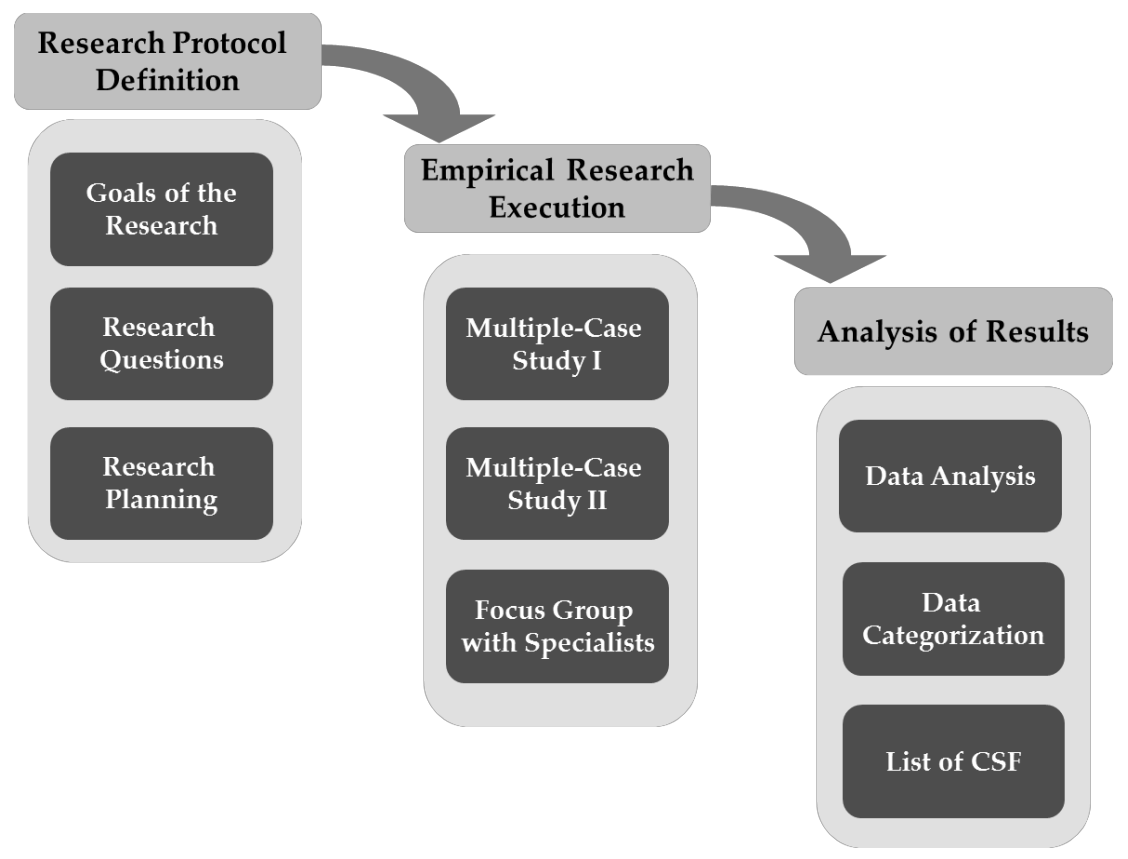

Figure 1. Research Phases

The qualitative approach allows the acquisition of novel knowledge and insights from empirical data. We adopted a non-probabilistic and purposeful sample selection strategy to define organizations and participants, as recommended by (Merriam, 2009). We considered the richness of the cases as our main selection criteria. A chain strategy (Merriam, 2009; Runeson \& Höst, 2008) was used and allowed participants to suggest other interesting cases and subjects.

As presented in Santos et al. (2011), RQ1 was answered through a literature review. This synthesis employed an ad-hoc approach, since no systematic method was adopted, and inclusions and exclusions of studies were not formally justified. Books, academic papers and practitioners' whitepapers were included in this literature review.

This initial literature survey was complemented by an initial case study (Multiple-Case Study I - MCS I). Our premise was that there were specific CSF for BPM initiatives in the public sector. With an exploratory emphasis, we identified barriers and facilitators experienced so far by four governmental organizations. 
Altogether, 20 practitioners participated in this case study, which employed semistructured interviews and focus groups as techniques for data gathering.

To obtain richer and more reliable answers to RQ2, we carried out an additional case study (Multiple-Case Study II - MCS II). This second study considered that there could be factors beyond those already provided by the initial case study. Eleven people from three public organizations took part in MCS II.

According to Yin (2009), MCS I and MCS II can be classified as holistic study of multiple-cases. This type has a single unit of analysis and investigates several cases. In our research, the units of analysis were the BPM initiatives, with public organizations as our cases. The protocol used in both case studies was carefully planned considering guidelines from (Yin, 2009).

By interviewing sponsors, leaders, process analysts, consultants and internal clients of BPM initiatives, we were able to create a list of CSF of BPM initiatives in the public sector. This was a central input to a discussion promoted with specialists to identify ways to manage these factors and enable their projects to succeed. This final study with BPM experts was conducted through a focus group section, where participants presented their opinions and contributed to enrich the analysis of each CSF presented. Finally, data was compiled and analyzed to determine key strategies to manage CSF of BPM initiatives.

As a strategy for data analysis in qualitative research, Flick (2009) suggests the use of specific procedures and techniques for text interpretation. We therefore transcribed and examined all interviews through a qualitative analysis of their content. They were then classified according to the following aspects: business domains of studied organizations, goals of BPM initiatives, methodological standards and, barriers and facilitators experienced along BPM initiatives execution. These categories supported data analysis and guided us to the results presented in Section 3.

\section{Results}

This section analyzes the results obtained for RQ3 - How to manage the critical success factors evidenced in BPM initiatives in the Public Sector? To answer this question, we performed a focus group. This session last for 1 hour and 48 minutes and involved five professionals with experience in BPM initiatives within the public sector. We present a brief profile of each expert as follows.

Expert 1 - IT consultant responsible for BPM projects in Brazilian Government. He has a master's degree in Computer Science and an MBA in Teaching in Higher Education. He has relevant experience in certification programs (ISO and CMM) and quality of software processes. He also taught several courses in BPM.

Expert 2 - he has about 20 years of professional experience in IT, organizational consulting and process management. He has a master's degree in Computer Science and he is currently undertaking a PhD research in BPM Governance. He has PMP (Project Management Professional) and CBPP (Certified Business Process Professional) certifications.

Expert 3 - he works for a Federal Public Organization that provides IT services for Brazilian Government. He was involved in the creation of the "BPM Platform", an 
initiative in partnership with the Brazilian Ministry of Planning. He was responsible for conceiving the "Process Modeling Methodology", the "Model of Management and Governance of the Processes Platform", among others. He has a master's degree in Computer Science, in the BPM area.

Expert 4 - since 1986, he works in a local Public Organization that provides IT services. He participated in the definition of business performance indicators, a project that later became focused on processes automation. He is currently involved in the implementation of a BPMS solution in a public organization.

Expert 5 - has five years of experience as project manager, being involved with BPM automation projects in diverse companies. He is currently acting as a BPM consultant, providing services for several public institutions.

At the beginning of the focus group, we presented the goals of the research to participants. Then, we presented the question to the discussion: What strategies can be performed to manage the identified CSF to increase the success chances of BPM initiative in the Public Sector?

Each critical success factor identified in MCS I and II was presented to the participants. We asked them to provide solutions for managing each CSF. When divergent opinions emerged, participants we allowed to complement their initial proposal and give additional suggestions. These strategies are following detailed.

Awareness of the benefits and challenges of BPM - The participants reported that despite the growing number of initiatives, the maturity of public organizations is still very low. Very often internal clients do not understand the notion of business process and perceive it as a legal process. This problem affects the results of BPM projects. To increase the participation of internal clients, they need to recognize the real benefits and requirements involved in BPM discipline. The following strategies were proposed by experts to address this challenge:

- Conduction of trainings, seminars and informal conversations before starting a BPM initiative: this efforts are a means to provide a better understanding of BPM goals and relevance, so that public servants engage in the project;

- Description of general impacts of the BPM practices within the organization: detailing the changes that shall occur in organizational structure and also the work procedures of each internal client.

BPM team composed by internal staff - This factor evidences that internal clients prefer BPM initiatives conducted by internal staff. Based on this, the participants suggested the strategies below:

- Definition of an internal core to support the BPM initiative: this unit can either be a Business Processes Management Office (BPMO) or a committee composed by members of strategic areas of the organization.

- This BPM team needs to establish and promote a common understanding of BPM initiative key goals. It must guarantee that all these objectives are available to internal clients; 
- Identify people interested and/or skilled on BPM: the BPM team must gather employees who demonstrate their intention to participate in the project and also those with background on BPM practices or experienced in improvement projects. The initiative sponsor may prove means to train this staff.

Bureaucracy and culture of the public sector - The Brazilian public sector is known for its slow and bureaucratic processes. To reduce this barrier and simplify the procedures in the public sector, business processes need to be reviewed. In this context, we should consider the following recommendations proposed by experts:

- To communicate the benefits and importance of BPM initiatives for internal clients, so that they can become allies to achieve the expected success;

- To emphasize that it is not beneficial to impose changes on the work of internal clients. The BPM team should involve them into the initiative in a natural way;

- To convince people from different sectors to actively interact and help to reduce the time imposed by bureaucracy;

- To clearly understand how the laws work and find legal alternatives that can improve the performance of these processes;

- To start with smaller processes that have a low impact on the organization and progressively increase the confidence of participants over time.

Communication between BPM team and internal staff - The way that BPM team communicates and keeps internal clients informed about the BPM initiative is a key factor that directly influences the success. Proposed strategies that can improve communication are:

- To create a website to promote the main achievements of the initiative and communicate its methodologies and general tools. This requires a complementary strategy to motivate internal clients or charge part of the BPM team to monitor website information;

- To structure a newsletter that can be sent by email or published in folders or bulletins;

- To conduct periodic internal workshops to disseminate the current results and communicate next steps and challenges. Over time, internal clients can improve their communication and better visualize the results.

Competences of the BPM team - The BPM team is characterized as the one responsible for conducting and institutionalizing the BPM initiative within the organization. Furthermore, it is recommended to the BPM team:

- To conduct periodic training to create disseminators of the initiative. These sessions should be followed by a performance assessment of participants. This may demonstrate their level of understanding of the subjects presents

- To perform a benchmark on BPM skills considered relevant by other public organizations. This will support the exchange of lessons learned; 
- To define the minimum competencies and skills that members must obtain for the BPM initiative.

Internal clients involvement with the BPM initiative - To attract internal clients in order to add value to the BPM initiative, specialists provided the following suggestions:

- The sponsor of the initiative must be engaged with awareness strategies. The sponsor should know that people need to be placed in their proper roles and responsibilities;

- Demonstrate the real benefits that the BPM initiative may bring to the organization;

- To establish a rewarding mechanism, so that internal clients can feel rewarded or charged in accordance with the objectives outlined;

- To inform internal clients what are the management priorities and engage them in these projects.

Internal staff experience with technological tools - Our research evidenced that staff is frequently unprepared on technological tools. Furthermore, internal customers generally neglect the relevance of new technologies for their activities. Hence, their ability to handle novel technologies may be a barrier to achieve the outcomes of the initiative. To manage this aspect it is fundamental to promote awareness and adequate training as a means to reduce the resistance of people to adopt novel tools.

Knowledge of internal staff on BPM concepts - To manage this factor, specialists provided the following suggestions:

- To provide BPM training to those involved in the initiative;

- To align the communication of BPM approaches to the reality of internal client involved in the initiative. In particular, it is important to adopt a common vocabulary to communicate concepts.

Impact of Government change due to elections - Due to political elections, strategies may change in public administration. To manage the impact caused by this factor, the following suggestions were made:

- To engage managers with roles and responsibilities clearly defined in the initiative, since directors and secretaries often change after elections;

- To determine project milestones for each government to reduce the impact of this change. When the project exceeds the government period, it becomes a challenge to keep the initiative with the same priority.

Impact of laws or internal rules - Legal issues often impact on planning and execution of BPM initiatives, causing delays and rework on projects.

- Participants considered that the relationship between the BPM team and the legal sector is paramount for projects to perform quickly and avoid unnecessary delays; 
- It is important to identify norms that affect business processes. The project sponsor must persuade the legal sector, which is generally more conservative.

Resources and technological infrastructure supporting the BPM initiative Managing this factor is simple but requires proper attention to avoid problems during the BPM initiative. Experts recommended identifying technological resources that are required for the initiative. These shall encompass basic features such as computers, internet services and software systems.

Support from senior management - The support of executive sponsors becomes almost indispensable when the project promotes changes in organizational structure that affects staff daily routines. To manage this factor in a BPM initiative, the following suggestions were proposed:

- To negotiate with the sponsor the objectives to be achieved, and also the roles and responsibilities of each actor in the project;

- To perform a critical analysis periodically to assess the execution of the plan. As the sponsor has specific expectations regarding the results of the BPM initiative, internal clients also need her support throughout the initiative.

Turnover of contractor staff - In Brazil, employees of public organizations are hired by public examination. Often employees do not have sufficient expertise in specific areas. Hence, it is a common practice to hire external staff. In particular, there is a high turnover of contractor staff. To deal with this factor, experts proposed the following suggestions:

- To keep an internal team focused on the goals of the BPM initiative;

- To establish an internal core of experts within the organization that will be responsible to support newcomers (e.g. an outsourced team).To document and share all the knowledge about BPM initiative through training for everyone involved in the initiative.

\section{Final Considerations}

\subsection{Discussion}

Although the understanding the critical success factors of BPM initiatives is accepted as an important issue by practitioners and academics, the identification and management of CSF is a topic that received little attention in the past. This becomes more evident when specific domains are investigated, such as the public sector. Due to high failure rates and an increasing investment in BPM initiatives, there is a general concern that success factors deserve special attention to achieve the goals of the initiative.

During the planning phase of the BPM initiatives, the public organizations should verify if any of the factors discovered by this research are applicable to their context. This helps defining appropriate strategies to handle each factor. Moreover, we have observed factors that were already identified by other studies, such as: lack of skilled people conducting the BPM initiative and internal clients with insufficient training on BPM concepts. In all investigated organizations we could observe the relevance of sponsorship from senior management, by means of financial and political support the BPM initiatives. 
Based on the list of CSF obtained in the case studies, a focus group was held with specialists in order to point out strategies to manage them. The main suggestion of the specialists to manage the CSF was to establish an internal group such as a Business Process Management Office (BPMO). Miers (2006) emphasizes that the establishment of a BPMO is a critical success factor for BPM initiatives. According to Paim et al. (2008), a BPMO can bring various benefits such as proper understanding of the processes, organizational development and improvement of communication, operation of integrated and coordinated activities.

\subsection{Limitations and Threats to Validity}

Despite of rigorous method adopted, this research has some limitations. Although case studies enable a wide analysis of variables selected within the domain of investigated organizations, this method is restricted to the particular situation analyzed. Hence, it is not possible to generalize the results obtained in this study for other organizations (Yin, 2009). Also, due to characteristics of semi-structured interviews, the analysis of results of this research is limited to what interviewees declared or remembered during interviews.

The applicability of the suggestions provided by experts to manage the CSF of BPM initiatives has not been scientifically tested in this research, i.e., we did not perform experiments to evaluate the effective use of proposed strategies. This is a limitation of the study that we aim to address in future research.

The threats to validity are discussed on the basis of three research questions, extending the evaluation presented in Santos et al. (2011). Based on Yin (2009), Table 1 presents four employed tests: case tests, recommended study tactics and also the study phase when the tactic should be applied.

Table 1. Studies tests and tactics

\begin{tabular}{|c|c|c|}
\hline Tests & Study Tactic & Phase of the research \\
\hline Construct validity & $\begin{array}{l}\text { - Use of multiple evidence sources } \\
\text { - Definition of evidence chain } \\
\text { - Results obtained by the researchers were reviewed } \\
\text { by the participants }\end{array}$ & $\begin{array}{l}\text {-Data collection } \\
\text {-Data collection } \\
\text {-Composition }\end{array}$ \\
\hline Internal validity & $\begin{array}{l}\text { - Not applicable to this research, since dependent } \\
\text { variables keep no causal relationship. }\end{array}$ & ---- \\
\hline External Validity & $\begin{array}{l}\text { - Use of multiple case studies theory } \\
\text { - Use of replication logic for multiple case studies } \\
\text { - Use of discussion with specialists }\end{array}$ & $\begin{array}{l}\text { - Research design } \\
\text { - Research design } \\
\text { - Research Execution } \\
\end{array}$ \\
\hline Reliability & $\begin{array}{l}\text { - Use of a research protocol and development of a } \\
\text { research planning } \\
\text { - Development of research documentation }\end{array}$ & $\begin{array}{l}\text {-Data collection } \\
\text {-Data collection }\end{array}$ \\
\hline
\end{tabular}

Construct validity focuses on whether the theoretical constructs are interpreted and measured correctly (Easterbrook et al. 2008). Yin (2009) indicates three tactics to increase construct validity. Following, we describe the strategies used in this study:

- Use multiple sources of evidence: interviews with sponsor and leader of the initiative, process analysts, internal clients and consultants who work or have worked in public organizations; 
- Establish chain of evidence: were performed different forms of data collection, such as individual interviews, focus groups and documentation research;

- Revision of participants: After the analysis and synthesis of the results, these were reviewed by the participants. If there was any disagreement, the investigator should adjust the results again. After we defined the list of FCS, it was reviewed by some participants in order to discuss the relevance of each factor.

Internal validity focuses on the study design, and particularly whether the results really do follow from the data (Easterbrook, 2008). Yin (2009) states that internal validity is only a concern for causal (or explanatory) case studies. Therefore, treatment of internal validity is outside the scope of this study.

External validity focuses on whether claims for the generality of the results are justified (Easterbrook, 2008). The criterion for selecting participants by convenience implies loss of external validity. However, it is not the purpose of this research to generalize the CSF of BPM initiatives in the public sector. Given that these factors may vary depending on the organizational structure, business area, department, office, among others.

Reliability focuses on whether the study yields the same results if other researchers replicate it. Yin (2009) discusses that the emphasis should be on doing the same case over again, instead of replicating the results of one case by doing another case study. In order to increase reliability, we developed a protocol for both multiple case studies. The protocol describes the procedures, techniques and objectives adopted. We adopted the same protocol to investigate all organizations (cases). Furthermore, the case study documentation was generated by adopting all the procedures described in the protocol.

\subsection{Future Research}

This paper aimed to analyze BPM initiatives within public organizations, focusing on identifying strategies to manage factors that may promote or hamper their success.

Several research opportunities may arise from the results of this study. In particular, we propose the following directions for further research:

- Conduction of additional empirical studies to evaluate the CSF identified in this research. Other research groups shall employ the same research protocol and apply a replication strategy to evaluate our findings. By comparing the results of the studies it is possible to present different CSF or to validate CSF identified in this research.

- Investigate the applicability of the strategies suggested by specialists to manage the CSF in BPM initiatives in the public organizations.

- Develop a BPM methodology for public organizations based on the results presented in this research. 


\section{References}

Aparecida da Silva, L., Damian, I. and Pádua, S. (2012) Process management tasks and barriers: functional to processes approach, Business Process Management Journal, Vol. 18, No. 5, pp. $762-776$.

ABPMP - Association of Business Process Management Professionals (2009) "BPM CBOK - Guide to the Business Process Management Common Body of Knowledge", vol 2.

Bandara, W., Gable, G. and Rosemann, M. (2005) Factors and measures of business process modelling: Model building through a multiple case study, European Journal of Information Systems, Vol. 14, No. 4, 347-360.

Burlton, R. (2011) BPM Critical Success Factors: Lessons Learned from Successful BPM Organizations, Business Rules Journal, Retrieved December 12, 2012 from http://www.brcommunity.com/b619.php.

Davidson, M. and Holt, R. (2008) "Failure Points: Where BPM Projects Tend To Falter". $\quad$ Retrieved October 20, 2010 from http://bpmmag.net/mag/failure_bpm_projects_1201/.

Flick, U. (2009) “An Introduction to Qualitative Research”, 4 ed., S. Netz, Ed., London: SAGE Publications.

FNQ - Fundação Nacional da Qualidade (2005) "Pessoas são a chave do sucesso", São Paulo: Classe Mundial.

Grover, V. (1999) From Business Reengineering to Business Process Change Management: A Longitudinal Study of Trends and Practices, IEEE Transactions on Engineering Management, Vol. 46, No. 1, 36-46.

Gulledge Jr, T. R. and Sommer, R. (2002) Business process management : public sector implications, Business Process Management Journal, Vol. 8, No. 4, 364-376.

Houy, C., Fettke P. and Loos, P. (2010) Empirical research in business process management - analysis of an emerging field of research, Business Process Management Journal, Vol. 16, No. 4, 619-661.

Jeston, J. and Nelis, J. (2008) "Business Process Management, practical guidelines to successful implementations", 2 ed., Oxford: Elsevier.

Ko, R., Lee, S. and Lee, E. (2009) Business process management (BPM) standards: a survey, Business Process Management Journal, Vol. 15, no. 5, pp. $744-791$.

Kotler, P. and Armstrong, G. (2009) "Principles of Marketing”, 13 ed., London: Prentice Hall.

Lock, M. (2008) "BPM and Beyond: The Human Factor of Process Management", November: Aberdeen Group.

McCoy, R. (2011) "Business Process Management (BPM) Key Initiative Overview", Retrieved November 11, 2011 from: http://www.gartner.com/DisplayDocument?id=1746423. 
Merriam, S. (2009) "Qualitative research: a guide to design and implementation", San Francisco: Jossey-Bass.

Miers, D. (2006) "The Keys to BPM Project Success". Retrieved Jully 20, 2011 from http://www.bptrends.com/publicationfiles/01-06-ART-KeysToBPMProjSuccess Miers.pdf.

OMG (2010) "Business Process Management with OMG specifications". ". Retrieved June 05, 2010 from http://www.bpm-consortium.org/literature.htm.

Paim, R., Caulliraux, H. and Cardoso, R. (2008) Process management tasks: a conceptual and practical reviews, Business Process Management Journal, Vol. 14, No. 5, 694-723.

Pritchard, J. and Armistead, C. (1999) Business process management - lessons from European business, Business Process Management Journal, Vol. 5, No. 1, 10-35.

Recker, M., Indulska, M., Rosemann and P. Green, (2009) Business Process ModelingA Comparative Analysis, Journal of the Association of Information Systems, Vol. 10, no. 4, pp. 333 - 363.

Runeson P. and Höst, M. (2008) Guidelines for conducting and reporting case study research in software engineering, Empirical Software Engineering, Vol. 14, No. 2, 131-164.

Santos, H., Santana, A. and Alves, C. (2011) Análise de Fatores Críticos de Sucesso da Gestão de Processos de Negócio em Organizações Públicas. VII Simpósio Brasileiro de Sistemas de Informação, 262-273.

Sentanin, O., Santos, F. and Jabbour, C. (2008) Business Process Management in a Brazilian Public Research Center. Business Process Management Journal, Vol. 14, No 4, 483-496.

Smith, G. and Furt, S. (2009) "How (not) to Fail at BPM". Retrieved May 25, 2012 from http://www.bpm.com/how-not-to-fail-at-bpm.html.

Trkman, P. (2010) The critical success factors of business process management" International Journal of Information Management, vol. 30, no. 2, 125-134.

M. Weske (2007) “Concepts, Languages, Architectures”, vol. 14, Berlin: SpringerVerlag.

Yin, R. (2009) "Case Study Research: Design and Methods", 4 ed., vol. 5, Sage Publications, Los Angeles. 